\title{
Une analyse rétrospective du début de l'épidémie de COVID-19 au Canada, du 15 janvier au 12 mars 2020
}

\author{
Équipe de surveillance et d'épidémiologie de la COVID-19 de l'Agence de la santé publique du \\ Canada ${ }^{1}$
}

\section{Résumé}

Description de l'épidémiologie de COVID-19 au début de l'épidémie au Canada, du 1er janvier au 12 mars 2020, avant que les gouvernements à tous les ordres ne mettent en œuvre des mesures de santé publique communautaires agressives. Au cours de cette période, 153 cas confirmés en laboratoire ont été signalés au Canada. En raison des retards inhérents au processus de diagnostic et de notification, ces cas représentaient un petit sous-ensemble des 1360 cas confirmés signalés par la suite, dont l'apparition des symptômes est survenue le 12 mars ou avant. Plus de la moitié (57,8 \%) de ces 1360 cas avaient des antécédents de voyages internationaux ou étaient liés à un cas qui avait voyagé, le plus souvent depuis des pays où peu de cas avaient été signalés à l'époque. La transmission communautaire, marquée par des cas qui ne pouvaient pas être rattachés à un autre cas, a été constatée pour la première fois le 20 février et a augmenté régulièrement par la suite. Cette analyse descriptive indique que la COVID-19 se répandait à l'échelle internationale et au Canada plus largement que ce qui avait été initialement détecté par les systèmes de surveillance de janvier à la mi-mars 2020. Afin de limiter l'impact des futures vagues, un système de surveillance élargi est actuellement mis en place avec de multiples flux de données afin de fournir une image plus complète de l'épidémie, y compris des signaux précoces de cas et de grappes. Un meilleur accès aux tests de laboratoire et une recherche élargie des contacts sont des éléments essentiels pour détecter et isoler les cas de manière précoce, y compris ceux qui présentent des symptômes légers.
Cette oeuvre est mise à la disposition selon les termes de la licence internationale Creative Commons Attribution 4.0

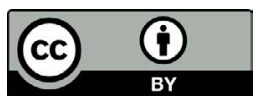

Affiliation

${ }^{1}$ Agence de la santé publique du Canada, Ottawa, ON

${ }^{\star}$ Correspondance :

lindsay.whitmore@canada.ca

Citation proposée : Équipe de surveillance et d'épidémiologie de la COVID-19 de l'Agence de la santé publique du Canada. Une analyse rétrospective du début de l'épidémie de COVID-19 au Canada, du 15 janvier au 12 mars 2020. Relevé des maladies transmissibles au Canada 2020;46(7/8):264-70.

https://doi.org/10.14745/ccdr.v46i78a04f

Mots-clés : épidémie, COVID-19, Canada, analyse rétrospective, épidémiologie

\section{Introduction}

À la suite de rapports faisant état de graves pneumonies d'étiologie inconnue à Wuhan, en Chine, en décembre 2019 et de la confirmation ultérieure que ces maladies étaient causées par un nouveau coronavirus le 7 janvier 2020, les gouvernements fédéral, provinciaux, territoriaux et locaux du Canada ont mis en place une surveillance accrue de la santé publique et ont pris des mesures de santé publique progressives pour prévenir la transmission de la maladie à l'intérieur de leurs frontières. Ces efforts comprenaient, entre autres, l'activation de plans intergouvernementaux d'intervention d'urgence en matière de santé publique, la mise en œuvre de mesures frontalières progressivement plus restrictives, ainsi que la mise en place et l'extension des capacités de dépistage. Les premiers efforts se sont concentrés sur le confinement à la source, afin de ralentir l'importation au Canada et d'empêcher la propagation à l'intérieur du pays.
Le premier cas importé du Canada a été signalé le 25 janvier 2020, mais ce n'est qu'à la mi-mars que le nombre de cas quotidiens a commencé à augmenter. Au 2 juin, 92151 cas avaient été signalés dans 12 provinces et territoires. Bien que le nombre de nouveaux cas quotidiens soit en baisse au moment de la rédaction du présent rapport, la maladie continue de se propager dans certaines régions et certains milieux, et il n'existe actuellement aucun vaccin contre ce nouveau coronavirus. Des mesures de santé publique fondées sur des données probantes sont essentielles pour contrôler l'épidémie au Canada. La compréhension de l'épidémiologie précoce de COVID-19 au Canada permettra de renforcer les systèmes de surveillance de la santé publique, de mettre en œuvre des mesures de santé publique appropriées et de mieux comprendre les impacts sur différents groupes (e.g. les femmes et les hommes, les différentes tranches d'âge). 
Cette communication rapide décrit l'épidémiologie de la COVID-19 au Canada du 1er janvier au 12 mars 2020. La date du 12 mars 2020 est importante, car elle marque le début de mesures de santé publique agressives mises en œuvre par les autorités fédérales, provinciales et territoriales pour limiter la transmission de la COVID-19 (e.g. fermeture d'écoles, fermeture d'entreprises non essentielles et contrôles stricts aux frontières) au Canada.

\section{Situation}

\section{Importation}

Sur la base des renseignements reçus des provinces et territoires au 17 avril 2020, 1360 cas confirmés en laboratoire ont été signalés avec une apparition de la maladie entre le $1{ }^{\text {er }}$ janvier et le 12 mars 2020. Les caractéristiques des cas sont résumées dans le tableau 1, y compris les cas par sexe et par âge. Au cours de cette période, plus de la moitié $(57,8 \%)$ des cas étaient liés à des voyages internationaux : $52,2 \%(n=710)$ avaient des antécédents de voyages internationaux et 5,6\% $(n=76)$ étaient liés à des cas qui avaient voyagé. Sur les 710 cas ayant un historique de voyages internationaux, 703 cas ont fourni des renseignements explicites sur les pays visités. Plus d'un tiers $(34,7 \%)$ de ces cas avaient transité par les États-Unis ou en étaient originaires, et près de $20 \%$ étaient arrivés du Royaume-Uni ou de France (tableau 2). Notamment, seulement $1,4 \%$ des cas étaient arrivés de Chine, alors que la Chine a été I'épicentre de la pandémie de COVID-19 tout au long des mois de janvier et février $(1,2)$.

Tableau 1 : Caractéristiques des cas de COVID-19 avec apparition de la maladie de janvier au 12 mars 2020 $(\mathrm{N}=1360)$

\begin{tabular}{|l|r|r|}
\hline \multirow{2}{*}{$\begin{array}{c}\text { Caractéristiques } \\
\text { du cas }\end{array}$} & \multicolumn{3}{|c|}{ Fréquence } \\
\cline { 2 - 3 } Province/territoire & $\mathbf{n}$ & \multicolumn{2}{c|}{} \\
\hline ON & 533 & 39,2 \\
\hline OC & 312 & 22,9 \\
\hline BC & 250 & 18,4 \\
\hline AB & 166 & 12,2 \\
\hline MB & 24 & 1,8 \\
\hline NS & 18 & 1,3 \\
\hline NB & 17 & 1,3 \\
\hline SK & 32 & 2,4 \\
\hline NL & 1 & 0,1 \\
\hline PE & 2 & 0,2 \\
\hline YT & 2 & 0,2 \\
\hline Voyageur rapatrié & 3 & 0,2 \\
\hline Sexe & & \\
\hline Femme & 670 & 49,5 \\
\hline Homme & 684 & 50,5 \\
\hline & & \\
\hline
\end{tabular}

Tableau 1 : Caractéristiques des cas de COVID-19 avec apparition de la maladie de janvier au 12 mars 2020 $(\mathrm{N}=1360)$ (suite)

\begin{tabular}{|l|r|r|}
\hline \multirow{2}{*}{$\begin{array}{c}\text { Caractéristiques } \\
\text { du cas }\end{array}$} & \multicolumn{3}{|c|}{ Fréquence } \\
\cline { 2 - 3 } Groupe d'âge (années) & $\mathbf{n}$ & \\
\hline $0-19$ & 52 & 3,9 \\
\hline $20-39$ & 368 & 27,8 \\
\hline $0-19$ & 52 & 3,9 \\
\hline $20-39$ & 368 & 27,8 \\
\hline $40-59$ & 510 & 38,5 \\
\hline $60-79$ & 341 & 25,8 \\
\hline 80 et plus & 53 & 4,0 \\
\hline $\begin{array}{l}\text { Hospitalisation (y } \\
\text { compris I'unité de } \\
\text { soins intensifs) }\end{array}$ & 195 & 17,5 \\
\hline Admissions à I'USI & 81 & 10,3 \\
\hline Décès & 27 & 2,5 \\
\hline
\end{tabular}

Abréviations : AB, Alberta; BC, Colombie-Britannique; MB, Manitoba; NB, Nouveau-Brunswick; NL, Terre-Neuve-et-Labrador; NS, Nouvelle-Écosse; ON, Ontario; QC, Québec;

SK, Saskatchewan; USI, unité de soins intensifs; YT, Yukon

a Les données manquantes concernant le sexe (six cas), l'âge (36 cas), l'hospitalisation (246 cas), les admissions à l'USI (573 cas) et les personnes décédées (280 cas) n'ont pas été incluses dans le calcul des pourcentages

${ }^{b}$ Les provinces et territoires peuvent définir le sexe différemment et certains peuvent se référe au sexe biologique

Tableau 2 : Pays visités par les cas de COVID-19 au Canada ayant déclaré un voyage international dans les 14 jours précédant l'apparition des symptômes, de janvier au 12 mars $2020(\mathrm{~N}=703)$

\begin{tabular}{|c|c|c|}
\hline Pays $^{\mathrm{a}, \mathrm{b}}$ & $\mathbf{n}$ & $\%$ \\
\hline États-Unis & 244 & 34,7 \\
\hline Royaume-Uni & 67 & 9,5 \\
\hline France & 64 & 9,1 \\
\hline Transport international (croisière) & 47 & 6,7 \\
\hline Allemagne & 45 & 6,4 \\
\hline Mexique & 35 & 5,0 \\
\hline Iran & 34 & 4,8 \\
\hline Égypte & 33 & 4,7 \\
\hline Espagne & 29 & 4,1 \\
\hline Suisse & 24 & 3,4 \\
\hline Autriche & 22 & 3,1 \\
\hline Portugal & 21 & 3,0 \\
\hline Philippines & 20 & 2,8 \\
\hline Cuba & 19 & 2,7 \\
\hline Pays-Bas & 19 & 2,7 \\
\hline Italie & 17 & 2,4 \\
\hline Chine & 10 & 1,4 \\
\hline
\end{tabular}


Les cas qui ont voyagé ont été catégorisés en fonction de la ou des régions (3) de l'Organisation mondiale de la santé (OMS) visitées afin d'examiner les tendances au fil du temps (figure 1). Au cours des trois premières semaines de l'épidémie (du 12 janvier au 26 janvier 2020), il y a eu sept cas et tous étaient soit des voyageurs arrivant de Chine (région du Pacifique occidental), soit leurs proches. Début février, quatre cas ont été signalés, avec un historique de voyage dans la région de la Méditerranée orientale, l'Europe et les Amériques. Des cas ayant des antécédents de voyage dans ces régions se sont produits malgré le petit nombre de cas signalés dans ces régions à l'époque [neuf cas dans la région de la Méditerranée orientale, 46 cas en Europe et 15 cas dans les Amériques (à l'exclusion du Canada)] (4). Au cours des deux dernières semaines de février, 99 cas au total ont transité par des pays d'Amérique ou en sont arrivés. Cela s'est produit malgré un total de seulement 65 cas signalés dans les pays des Amériques (à l'exclusion du Canada) à la fin du mois de février (2).

Un certain nombre de mesures sanitaires aux frontières et en voyage ont été mises en œuvre au cours de cette période (i.e. conseils de santé aux voyageurs, amélioration du dépistage des symptômes et messages de santé publique aux points d'entrée et demande aux voyageurs internationaux arrivant de certaines régions de s'isoler pendant 14 jours). La mise en œuvre des mesures spécifiques à chaque pays a été guidée, en partie, par le nombre de cas de COVID-19 et le type de transmission qui a été déclaré par l'OMS et les divers pays. Le type de transmission a été classé par l'OMS en tant que cas importés uniquement, transmission locale (la transmission s'est produite à l'intérieur du pays avec quelques cas non liés à des chaînes de transmission connues) ou transmission communautaire, définie comme l'incapacité de relier un grand nombre de cas confirmés à des chaînes de transmission (2). À l'époque, à l'exception de la Chine, tous les cas importés au Canada provenaient de pays évalués par l'OMS comme des pays où il n'y avait que des cas importés ou une transmission locale sans transmission communautaire. En rétrospective, il est clair que ce qui a été rapporté à l'échelle mondiale n'était que la partie émergée de l'iceberg et que la véritable étendue de la propagation mondiale de la maladie n'était pas connue à l'époque. Sur la base des enseignements tirés de la première vague de COVID-19, des sources de données et des indicateurs supplémentaires seront inclus dans les évaluations des risques qui sont utilisées pour informer diverses mesures sanitaires aux frontières et en voyage, comme l'affichage en temps utile des avis sanitaires aux voyageurs.

\section{Détection précoce}

La détection précoce des cas est essentielle pour mettre en œuvre des mesures de santé publique efficaces à tous les ordres de gouvernement (local, provincial, territorial et fédéral). Au début de l'épidémie, le nombre médian de jours entre l'apparition de la maladie et la date de déclaration des cas (tel que constaté par les autorités de santé publique locaux) différait sensiblement selon le type d'exposition $(p<0,05)$. Les cas liés à des voyages internationaux et ceux qui étaient liés à un cas connu avaient un nombre médian de jours légèrement inférieur

Figure 1 : Cas de COVID-19 confirmés en laboratoire au Canada selon la date d'apparition de la maladie et la région de voyage pour les cas signalant un voyage international, du 15 janvier au 12 mars 2020 ( $N=1275)$

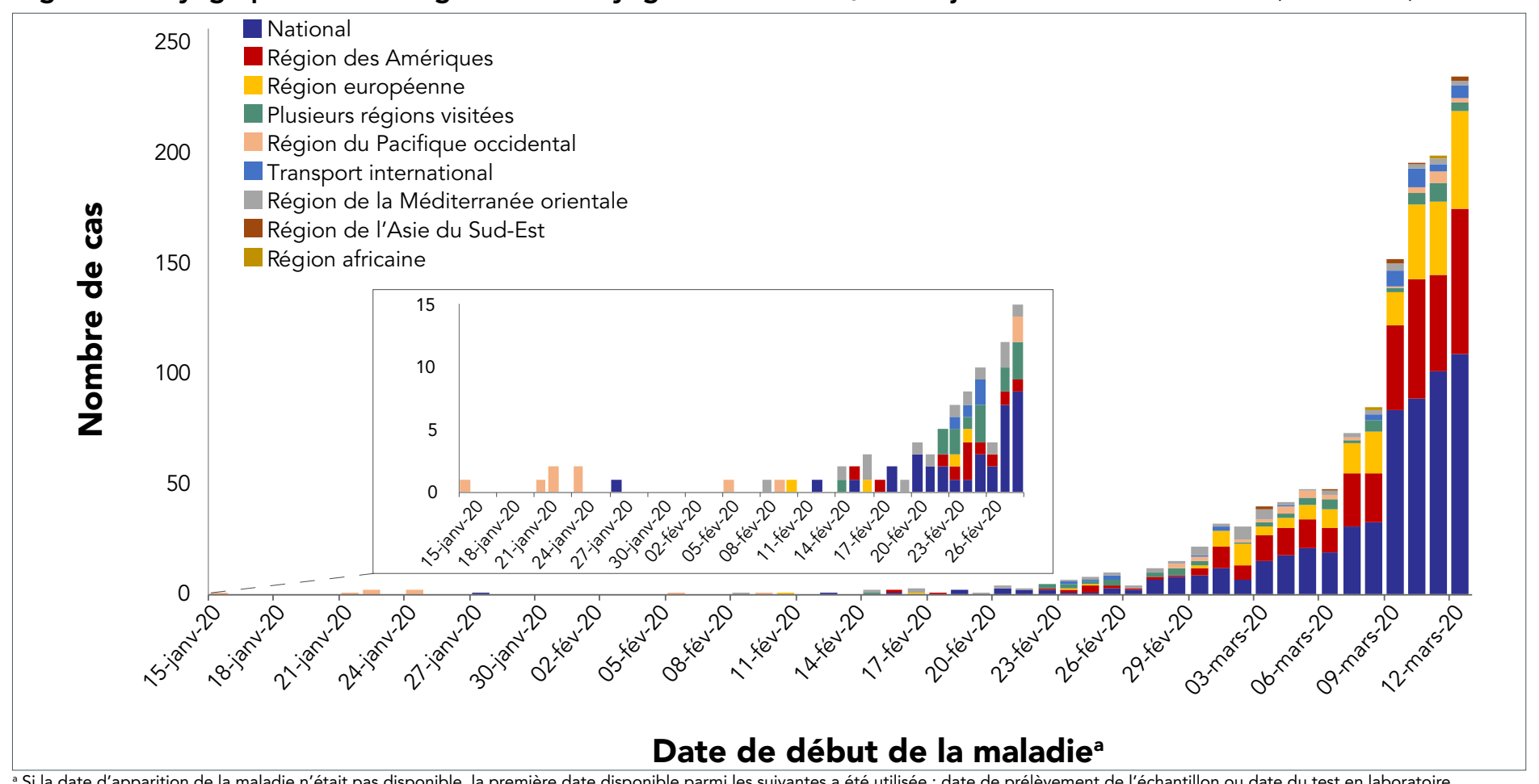


entre l'apparition de la maladie et la déclaration par rapport aux cas qui ne pouvaient pas être liés à un cas connu (tableau 3 ). Dans d'autres pays, des différences ont été constatées entre ceux dont les infections ont été contractées à l'étranger et ceux dont les infections ont été contractées au pays $(5,6)$. Ces différences au Canada et ailleurs étaient probablement dues à un indice de suspicion plus élevé pour ceux dont les facteurs de risque étaient connus à l'époque. Le nombre de jours entre le début de la maladie et la date de déclaration du cas a augmenté au fil du temps, car le nombre de cas qui ne pouvaient pas être liés à d'autres cas a également augmenté. Ce délai a commencé à diminuer pour les cas dont la maladie est apparue début mars (figure 2).

Tableau 3 : Nombre de jours entre la date d'apparition de la maladie et la date de déclaration du cas $(N=727)^{a}$

\begin{tabular}{|c|c|c|c|c|}
\hline $\begin{array}{c}\text { Catégorie } \\
\text { d'exposition }\end{array}$ & $\mathbf{n}$ & $\begin{array}{l}\text { Nombre } \\
\text { médian } \\
\text { de jours }\end{array}$ & $\begin{array}{c}25^{\mathrm{e}} \\
\text { percentile }\end{array}$ & $\begin{array}{c}75^{e} \\
\text { percentile }\end{array}$ \\
\hline $\begin{array}{l}\text { Antécédents } \\
\text { de voyages } \\
\text { internationaux }\end{array}$ & 400 & 9 & 6 & 15 \\
\hline $\begin{array}{l}\text { Contracté au pays- } \\
\text { Lié à un voyageur }\end{array}$ & 49 & 7 & 5 & 11,5 \\
\hline $\begin{array}{l}\text { Contracté au pays- } \\
\text { Lié à un cas national }\end{array}$ & 134 & 8 & 6 & 14 \\
\hline $\begin{array}{l}\text { Contracté au pays- } \\
\text { N'a pas pu être lié }\end{array}$ & 87 & 11 & 10 & 18 \\
\hline $\begin{array}{l}\text { National- } \\
\text { Coordonnées non } \\
\text { spécifiées }\end{array}$ & 57 & 11 & 8 & 19 \\
\hline
\end{tabular}

Figure 2 : Nombre de jours entre la date de l'apparition de la maladie et la date de déclaration du cas (moyenne mobile de sept jours) pour les cas de COVID-19 par exposition, du 15 janvier au 12 mars $2020(N=727)$

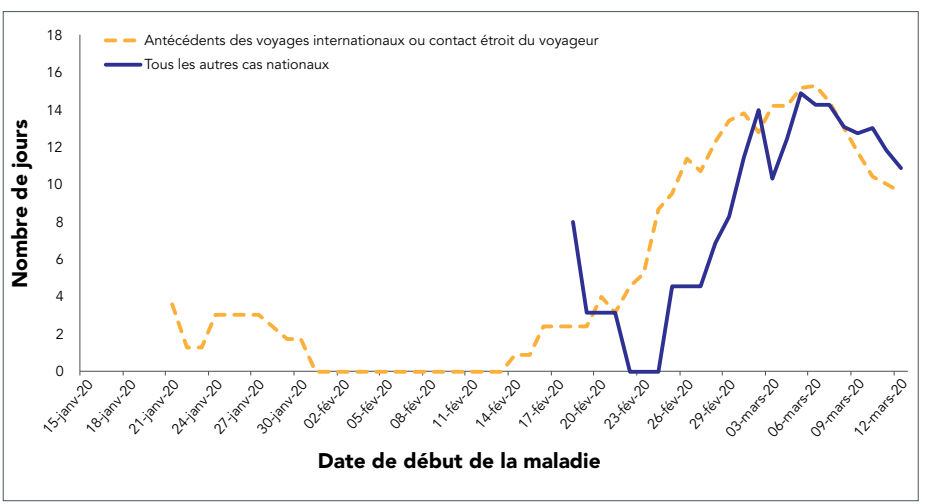

Aux niveaux provincial, territorial et fédéral, des renseignements opportuns sur les cas sont nécessaires pour élaborer, mettre en œuvre et évaluer les programmes et les politiques visant à limiter la transmission. En raison du délai entre l'apparition de la maladie et la déclaration des cas, les provinces et territoires n'avaient déclaré que 153 cas de COVID-19 en date du 12 mars, contre 1360 cas confirmés en laboratoire avec apparition réelle de la maladie pendant cette période (voir figure 3 ). De plus, il y a probablement eu des infections non déclarées pendant cette période en raison du dépistage limité effectué à l'époque. Les résultats obtenus dans certains pays ont indiqué que la maladie se propageait déjà avant que l'augmentation rapide des cas ne soit apparente et constatée, ou, dans le cas de la France, avant même que le premier cas ne soit signalé $(7,8)$.

\section{Figure 3 : Nombre de cas de COVID-19 signalés quotidiennement par les provinces et territoires par rapport aux cas d'apparition de la maladiea, du 15 janvier au 12 mars 2020}

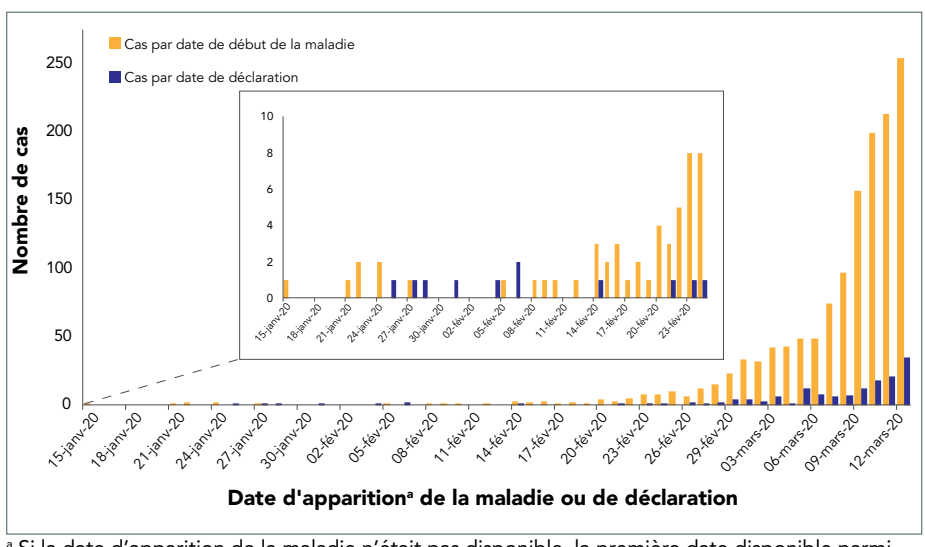

Si la date d'apparition de la maladie n'était pas disponible, la première date disponible parmi les suivantes a été utilisée : date de prélèvement de l'échantillon et date du test en laboratoire

Au début de l'épidémie, les efforts de surveillance de la santé publique ont été guidés par les directives de surveillance du Plan canadien de lutte contre la pandémie d'influenza (9). Par exemple, dans les premiers stades de l'épidémie au Canada, des renseignements détaillés ont été recueillis sur les cas confirmés (10) pour permettre une description des caractéristiques épidémiologiques des cas. Ces résultats ont été complétés par des efforts provinciaux et territoriaux, notamment une surveillance accrue dans certains milieux (e.g. le dépistage des personnes souffrant de maladies respiratoires dans les hôpitaux). À l'avenir, un certain nombre de flux de données complémentaires seront intégrés, afin de contribuer à renforcer les efforts de surveillance dans le cadre de la COVID-19 au niveau national et de détecter les premiers signes d'augmentation du nombre de cas ou de grappes, ou d'évolution dans la gravité de la maladie. Ces flux de données devraient inclure, entre autres, des renseignements provinciaux et territoriaux fondés sur des cas, des données de laboratoires de santé publique fédéraux et provinciaux, des données sur les hôpitaux, les urgences et les patients ambulatoires, des renseignements sur les épidémies et la surveillance syndromique grâce à l'application «ActionGrippe » (11). Les résultats des études futures seront utilisés pour exploiter les renseignements recueillis dans le cadre de ces activités de surveillance et fourniront des renseignements plus détaillés sur les populations 
clés (par exemple, l'impact de la COVID-19 sur les communautés racialisées, les travailleurs de la santé, les communautés autochtones et divers groupes de femmes, d'hommes et de personnes de diverses identités de genre).

\section{Retransmission}

Au cours des quatre premières semaines de l'éclosion au Canada (du 12 janvier au 2 février), $100 \%$ des cas étaient liés à un voyage (avaient voyagé ou étaient liés à un voyageur). Au cours de la période du 9 février au 12 mars 2020, entre $11 \%$ et $40 \%$ des cas, par semaine, étaient des cas nationaux qui pouvaient être rattachés à un autre cas national. Le 23 février, le premier cas est tombé malade et n'a pas pu être relié à un autre cas connu (un indicateur de transmission communautaire), après quoi le nombre de ces cas a augmenté régulièrement (figure 4). D'autres pays ont également connu une courte phase de cas essentiellement importés, suivie par l'apparition et la croissance exponentielle de cas contractés au pays (12).

Pour prévenir la retransmission, au début de l'épidémie, des lignes directrices sur la prévention et le contrôle de l'infection et sur la gestion des cas et des contacts par la santé publique ont été diffusées aux établissements et aux professionnels de la santé publique. Les Canadiens ont été invités à surveiller eux-mêmes leurs symptômes, à rester chez eux lorsqu'ils sont malades et à prendre des mesures d'hygiène fréquentes et méticuleuses. Néanmoins, la transmission communautaire s'est produite et le nombre de ces cas a rapidement augmenté. Cela peut refléter un certain nombre de facteurs : le Canada était encore dans sa saison grippale et il se peut que des personnes n'aient pas demandé de dépistage si elles présentaient des symptômes de type grippal, la contribution de la transmission par des personnes présentant des symptômes légers qui n'ont pas demandé de dépistage ou qui n'étaient pas admissibles à un dépistage compte tenu des directives relatives au dépistage en vigueur à l'époque, ou la transmission par des personnes qui étaient pré- ou asymptomatiques (13).

\section{Conclusion}

Au début de l'épidémie au Canada, les mesures de santé publique visant à limiter la propagation de la COVID-19 ont été guidées, en partie, par les données épidémiologiques disponibles à l'époque. Rétrospectivement, il est clair que ces données n'ont pas permis de dresser un tableau complet de la situation en ce qui concerne le moment et le lieu où la maladie s'est propagée ni de savoir comment et quand des individus ont pu transmettre l'infection à d'autres. Au fur et à mesure que de nouvelles données sont apparues pour élargir nos connaissances sur l'épidémiologie de la COVID-19, nos protocoles de détection des cas, les mesures de santé publique et d'autres domaines de la réponse à la COVID-19 ont été adaptés. À l'avenir, les systèmes de surveillance continueront également à être améliorés par l'ajout de nombreux flux de données, ce qui permettra d'obtenir une image plus complète de l'épidémiologie de COVID-19 et de détecter rapidement les cas et les grappes afin d'informer les mesures de santé publique et d'améliorer la gestion de la COVID-19 au Canada.

Figure 4 : Cas de COVID-19 confirmés en laboratoire au Canada par date d'apparition de la maladie et par catégorie d'exposition, du 15 janvier au 12 mars $2020(N=1276)$

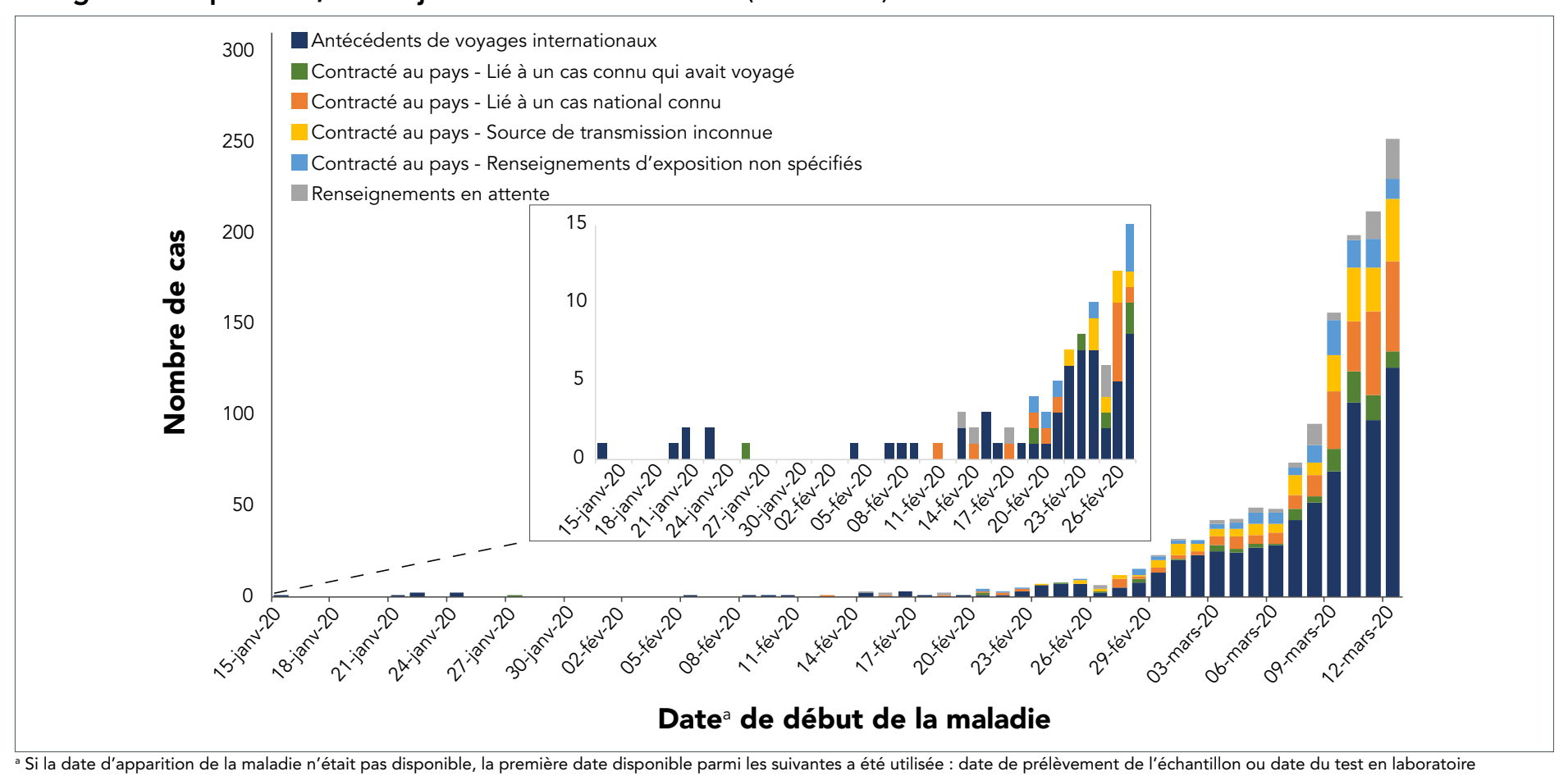




\section{Déclaration des auteurs}

D. P. - Conceptualisation, ébauche originale, révision et correction

L. L. - Conservation des données, analyse formelle, révision et correction

L. W. - Révision et correction

A. C. - Conceptualisation, révision et correction

K. W. - Révision et correction

M. B. - Révision et correction

D. T. - Révision et correction

C. A. - Révision et correction

J. T. - Conservation des données, analyse formelle

J. Z. - Conservation des données, analyse formelle

J. K. - Conservation des données, analyse formelle

D. M. - Révision et correction

A. N. - Révision et correction

J. P. - Révision et correction

\section{Intérêts concurrents}

Aucun.

\section{Remerciements}

Les auteurs tiennent à remercier les partenaires de surveillance provinciaux et territoriaux, le Laboratoire national de microbiologie et les laboratoires de santé publique provinciaux, ainsi que l'équipe de surveillance du Centre des opérations du portefeuille de la Santé de l'Agence de la santé publique du Canada.

L'équipe de surveillance et d'épidémiologie de la COVID-19 de l'Agence de la santé publique du Canada comprend les personnes suivantes: Dana Paquette, Liza Lee, Lindsay Whitmore, Andrea Currie, Kerri Watkins, Maggie Bryson, Dorcas Taylor, Chris Archibald, Jill Tarasuk, Jingxuan Zhang, Jaskiran Kaur, Diane MacDonald, Alexandra Nunn, Jennifer Pennock.

\section{Financement}

Ce travail a été soutenu par l'Agence de la santé publique du Canada.

\section{Références}

1. World Health Organization. Novel Coronavirus (2019nCoV) Situation Report - 1: 21 January 2020. Geneva (CH): WHO (accédé 2020-05-19). https://www.who.int/docs/ default-source/coronaviruse/situation-reports/20200121sitrep-1-2019-ncov.pdf?sfvrsn=20a99c10_4

2. World Health Organization. Novel Coronavirus (2019-nCoV) Situation Report - 40: February 29, 2020. Geneva (CH): WHO (accédé 2020-05-19). https://www.who.int/docs/ default-source/coronaviruse/situation-reports/20200229sitrep-40-covid-19.pdf?sfvrsn=849d0665_2
3. Organisation mondiale de la Santé. Bureaux régionaux de I'OMS (accédé 2020-05-19). https://www.who.int/fr/about/ who-we-are/regional-offices

4. World Health Organization. Novel Coronavirus (2019-nCoV) Situation Report - 26: 15 February 2020. Geneva (CH): WHO (accédé 2020-05-19). https://www.who.int/docs/ default-source/coronaviruse/situation-reports/20200215sitrep-1-2019-ncov.pdf?sfvrsn=20a99c10_4

5. Spiteri G, Fielding J, Diercke M, Campese C, Enouf V, Gaymard A, Bella A, Sognamiglio P, Sierra Moros MJ, Riutort AN, Demina YV, Mahieu R, Broas $M$, Bengnér $M$, Buda S, Schilling J, Filleul L, Lepoutre A, Saura C, Mailles A, Levy-Bruhl D, Coignard B, Bernard-Stoecklin S, Behillil S, van der Werf S, Valette M, Lina B, Riccardo F, Nicastri E, Casas I, Larrauri A, Salom Castell M, Pozo F, Maksyutov RA, Martin C, Van Ranst M, Bossuyt N, Siira L, Sane J, Tegmark-Wisell K, Palmérus M, Broberg EK, Beauté , Jorgensen P, Bundle N, Pereyaslov D, Adlhoch C, Pukkila J, Pebody R, Olsen S, Ciancio BC. First cases of coronavirus disease 2019 (COVID-19) in the WHO European Region, 24 January to 21 February 2020. Euro Surveill 2020;25(9):2000178. DOI PubMed

6. Kwok KO, Wong VW, Wei WI, Wong SY, Tang JW. Epidemiological characteristics of the first 53 laboratory-confirmed cases of COVID-19 epidemic in Hong Kong, 13 February 2020. Euro Surveill 2020;25(16):2000155. DOI PubMed

7. Valenti L, Bergna A, Pelusi S, Facciotti F, Lai A, Tarkowski M, Berzuini A, Caprioli F, Santoro L, Basellli G, Ventura CD, Erba E, Bosari S, Galli M, Zehender G, Prati D. SARS-CoV-2 seroprevalence trends in healthy blood donors during the COVID-19 Milan outbreak. https://www.medrxiv.org/content /10.1101/2020.05.11.20098442v1.full.pdf

8. Deslandes A, Berti V, Tandjaoui-Lambotte Y, Alloui C, Carbonnelle E, Zahar JR, Brichler S, Cohen Y. SARS-CoV-2 was already spreading in France in late December 2019. Int J Antimicrob Agents 2020;55(6):106006. DOI PubMed

9. Réseau pancanadien de santé publique. Préparation du Canada en cas de grippe pandémique : Guide de planification pour le secteur de la santé Annexe traitant de la surveillance. Ottawa (ON) : ASPC; le 3 décembre 2015 (accédé 2020-05-19). https://www.canada.ca/content/dam/ phac-aspc/migration/phac-aspc/cpip-pclcpi/assets/pdf/ annex_n-fra.pdf

10. Gouvernement du Canada. Lignes directrices nationales provisoires en matière de surveillance de l'infection humaine par la maladie à coronavirus (COVID-19). Ottawa (ON) : Gouvernement du Canada (modifié 2020-02-10; accédé 2020-05-21). https://www.canada.ca/fr/sante-publique/ services/maladies/2019-nouveau-coronavirus/ directives-provisoires-surveillance-infection-humaine.html

11. Gouvernement du Canada. ActionGrippe. Ottawa (ON) : Gouvernement du Canada (modifé 2020-03-31; accédé 2020-05-27). https://www.canada.ca/fr/sante-publique/ services/maladies/grippe-influenza/grippe-a-loeil.html 
12. Weber A, lannelli F, Goncalves S. Trend analysis of the COVID-19 pandemic in China and the rest of the world. https://arxiv.org/pdf/2003.09032.pdf
13. Hu Z, Song $C, X u$ C, Jin G, Chen $Y, X u X, M a H$, Chen $W$ Lin Y, Zheng Y, Wang J, Hu Z, Yi Y, Shen H. Clinical characteristics of 24 asymptomatic infections with COVID-19 screened among close contacts in Nanjing, China. Sci China Life Sci 2020 May;63(5):706-11. DOI PubMed

\section{Gratmit! publique du Canada Agency of Canada \\ MATÉRIEL DE SENSIBILISATION À LA VACCINATION}

Disponible dans les deux langues officielles
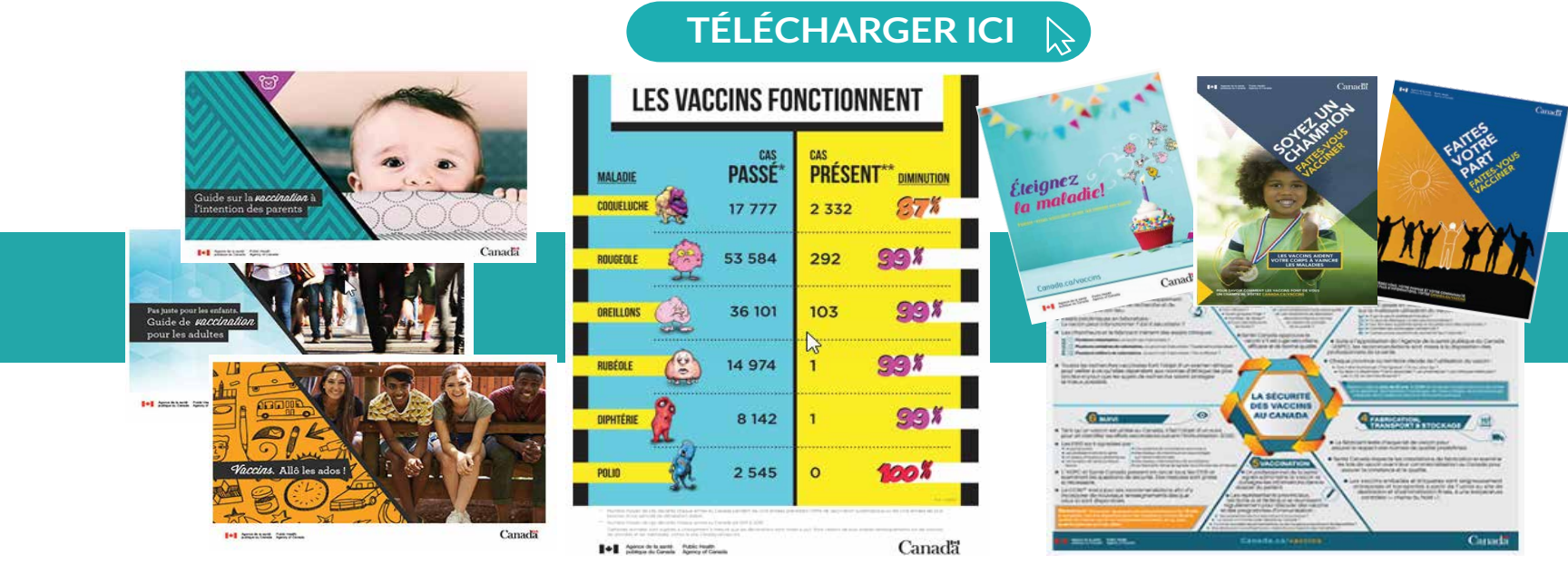

Pour plus d'informations sur les vaccins et la vaccination visitez:

\section{Canada.ca/vaccins}

